\title{
The Effect of Variable Spontaneous Curvature on Dynamic Evolution of Two-Phase Vesicle
}

Mohammad Mahdi Sahebifard* ${ }^{*}$ Alireza Shahidi and Saeed Ziaei-Rad

Department of Mechanical Engineering, Isfahan University of Technology, Isfahan, Iran

\begin{abstract}
This article aims to study the effect of non-uniform distribution of spontaneous curvature on shape transformation of two-phase vesicles via an evolutionary method. Their dynamic evolution is developed based on conventional Helfrich theory, considering bending of the membrane and friction in the surrounding fluid in each phase with variable spontaneous curvature. The variation of spontaneous curvature is assumed to be a function of arc length in each domain considering the effects of inducing factors (surrounding solution concentration and the membrane-protein interactions such as scaffolding and insertion). Membrane pearling from a large vesicle is simulated by the model and compared with the result of constant curvature and also with empirical observations. It can be shown that accurate simulation of some membrane deformation mechanisms depends on careful consideration of key factors such as the SC variations. In addition, the importance of different uniform and non-uniform distributions of spontaneous curvature is discussed with reference to specific cases.
\end{abstract}

Keywords: Two-phase vesicle; Evolutionary model; Variable spontaneous curvature; Protein distribution; Membrane pearling

\section{Introduction}

Lipid bilayer membranes consist of polar molecules which are positioned across the membrane and any deflection in their normal arrangement requires a consumption of energy. These membranes are naturally symmetric and have no orientation. However, some factors like proteins can locally change the bilayer structure arrangement, resulting in curvature induction to the membrane [1]. The effects of spontaneous curvature (SC) on studying behavior of lipid membranes have been considered in many theoretical and experimental researches [2-4].

Although SC is considered as an invariable parameter in common known continuum models of vesicles [5,6], non-uniform distribution of curvature is a usual phenomenon [7,8]. Reference [9] presents an equilibrium model of a flat membrane with variable SC from the perspective of elastic shell theory. The effect of variable SC on local tension was also studied for a two-dimensional flat membrane [10]. An [11] equilibrium model is developed regarding variable curvature in which SC is considered as a function of polymer concentration gradient. Also in uniform vesicles, variable SC has been surveyed using local changes in molecular density [12]. The present study introduces an axisymmetric evolutionary model of two-phase vesicles with variable SC. Two-phase vesicles as examples of heterogeneous vesicles have a better efficiency to simulate closed membranes.

Several factors may cause SC in lipid membranes (for example, different molecular properties of the constituent membrane lipids, mechanisms by which proteins can generate membrane curvature such as scaffolding and insertion and local concentrations of adsorbing particles). Using the model, the effect of these mechanisms can be considered for more accurate simulation.

In the previous paper, the dynamic evolution of two-component vesicle has been developed using the equilibrium between the membrane bending potential and local fluid friction in each phase. Also, stationary and evolutionary methods were compared and stated that evolutionary method is a generalized form and evaluation criteria for related stationary/equilibrium model [13]. In this study, firstly equations dominating each phase of the membrane are presented considering variable SC. Then regarding the matching conditions of two domains, updated expressions of the membrane section reactions are introduced. Finally the model is described for some specific cases. By examining some examples of curvature distribution, the importance of this factor on shape transformation of vesicle membranes is highlighted. Next, a giant vesicle is simulated under the influence of protein insertion, with protein insertion into a small area of the membrane assumed to be a hyperbolic function with significant changing values and slope. In our results, budding and pearling processes are observed similar to experimental observations. We also show that it is not easy to simulate the pearling process with constant SC.

\section{Modeling}

\section{Evolutionary equation}

The current study considers a lipid bilayer axisymmetric closed membrane consisting of two separate phase. $r$ and $\theta$ are polar coordinates in the $x-y$ plane and s represents the measured arc length from the south pole of the vesicle, $z=0$ (Figure 1). Assuming constant bending rigidity of the membrane, $k$ the elastic free energy of the membrane, $E_{B}$, is proportional to an integral of the square of the membrane curvature, $\tau$ : [5]

$$
E_{B}=\frac{1}{2} k \int_{\Gamma}^{2} d A(H-C(s))^{2}
$$

In which $H=2 h=\psi+\frac{\sin \psi}{r}$ indicates total curvature and $C(s)$ represents SC of the membrane. Variable SC is considered as a function of geometry and arc length that can be specified by different methods such as experimental data or molecular dynamics [2-4].

*Corresponding author: Mohammad Mahdi Sahebifard, Department of Mechanical Engineering, Isfahan University of Technology, Isfahan, Iran, Tel: 8415683111; E-mail: MM.Sahebifard@me.iut.ac.ir

Received June 15, 2017; Accepted June 21, 2017; Published June 23, 2017

Citation: Sahebifard MM, Shahidi A, Ziaei-Rad S (2017) The Effect of Variable Spontaneous Curvature on Dynamic Evolution of Two-Phase Vesicle. J Adv Chem Eng 7: 175. doi: 10.4172/2090-4568.1000175

Copyright: @ 2017 Sahebifard MM, et al. This is an open-access article distributed under the terms of the Creative Commons Attribution License, which permits unrestricted use, distribution, and reproduction in any medium, provided the original author and source are credited. 


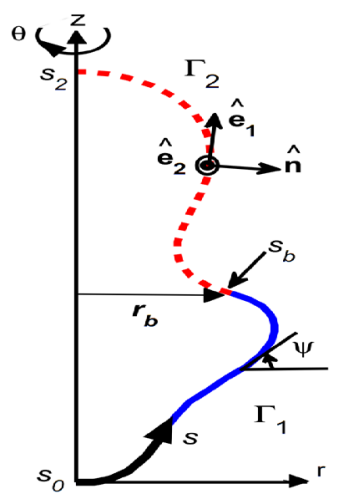

Figure 1: Coordination of $r$ and $z$ in definition of axisymmetric geometry along with the position of orthonormal basis in each point of surface which are defined in index $b$ refers to the boundary.

The evolutionary model of the membrane is presented for a two-phase vesicle based on a local equilibrium between the bending potential force of the membrane and fluid friction [13]:

$$
k v=-\frac{\delta E_{B}}{\delta \Gamma}-P+\tau H
$$

Assuming variable SC and $(C(s))^{\prime}=\frac{d}{d s}$.

$$
\frac{\delta E_{B}}{\delta \Gamma}=k^{(i)}\left[\begin{array}{l}
\left(H^{(i)}-(C(s))^{(i)}\right)^{\prime}+\left(H^{(i)}-(C(s))^{(i)}\right)^{\prime} \frac{\cos \psi}{r} \\
+\left(H^{(i)}-(C(s))^{(i)}\right)\left(\left(H^{(i)}\right)^{2}-2 k^{(i)}\right)-\frac{1}{2}\left[\left(H^{(i)}-(C(s))^{(i)}\right)^{2} H^{(i)}\right]
\end{array}\right]
$$

In which $v$ and $K=\psi^{\prime} \frac{\cos \psi}{r}$ denotes membrane normal velocity and Gaussian curvature, respectively. Lagrange multipliers $P$ and $\tau$ are used for imposing constraints of volume and area on the evolutionary equation. Parameter $P$ reveals the difference between interior and exterior pressure of the membrane, $(i)$ represents the negative value of surface tension in each domain of the two-phase vesicle. Finally, differential equations dominating in each phase are as below:

$k\left[-\frac{d r}{d t} \sin \psi+\frac{d z}{d t} \cos \psi\right]=k^{(i)}\left[\begin{array}{l}\left(H^{(i)}-(C(s))^{(i)}\right)^{\prime}+\left(H^{(i)}-(C(s))^{(i)}\right)^{\frac{\cos \psi}{r}} \\ +\frac{1}{2}\left(H^{(i)}-(C(s))^{(i)}\right)\left(\left(H^{(i)}\right)^{2}-4 k^{(i)}\right)+\left[\left(H^{(i)} C^{(i)}\right)\right]-P+\tau^{(i)} H^{(i)}\end{array}\right]$

Two helpful dimensionless parameters to describe the vesicles configuration are the area fraction of each phase $x^{(i)}=\frac{A(i)}{4 \pi R_{0}^{2}}$, and the reduced volume, $v=V /\left(\frac{4 \pi}{3} R_{0}^{3}\right)$, such that $R_{0}$ stands for radius of a sphere whose surface area is equivalent to the total area of the vesicle phases $\left(A^{(1)}+A^{(2)}=4 \pi R_{0}^{2}\right)$.

\section{Matching conditions}

Assuring equilibrium of phases requires determination of conditions at the matching area between phases. According to the method presented by Baumgart [14], we must set matching conditions:

$$
\begin{aligned}
& T_{s}^{s^{-}}-T_{s}^{s^{+}}-\sigma \frac{\cos \psi}{r}=0 \\
& Q_{s}^{+}-Q_{s}^{-}-\sigma \frac{\sin \psi}{r}=0 \\
& M_{s}^{s+}-M_{s}^{s-}=0
\end{aligned}
$$

In which, $T_{S}^{S}, Q_{S}$ and $M_{S}^{S}$ indicate lateral tension stress, transverse shear and bending moment correspondingly in each phase. Regarding variable SC, they are presented as below:

$$
\begin{aligned}
& T_{s}^{s}=k(H-C(\mathrm{~s}))\left[\psi^{\prime}-\frac{(H-C(\mathrm{~s}))}{2}\right]-\tau \\
& Q_{s}=k(H-C(\mathrm{~s}))^{\prime} \\
& M_{s}^{s}=k(H-C(\mathrm{~s}))+k_{G} \frac{\sin \psi}{r}
\end{aligned}
$$

Signs of - and + represnt just before and after the matching boundary. Numerical solution of the differential equations was carried out by developing calculation code in MATLAB and using bvp4s solver. For more information about the method of model solution see [13].

\section{Curvature distribution function}

In the current article, curvature distribution is studied by combinations of $\boldsymbol{t a n h}$ functions which help to determine step changes with low or high slope. The function of dimensionless SC, $\hat{C}^{(i)}=C^{(i)} R_{0}$ , is as follows:

$$
\hat{C}^{(i)}=A(1-\tanh (a S-b \pi))+c
$$

This function is used as an appropriate presentation for strong conversion of protein density from a constant value to zero between surfaces covered and uncovered by protein [15]. For constant values of curvature, A, the occupied surface is defined using as a parameter the central angle, $S$, in an equivalent sphere. $S$ denotes the central angle variable and corresponds to the arc length, $s$; their relationship is described as $\frac{d s}{d S}=\frac{\sin S}{r}$. In each phase, $\mathrm{S} \in[0, \pi]$, so that $S_{1}\left(s_{0}\right)=S_{2}\left(s_{2}\right)=0$ corresponds to the pole of that phase and at the matching boundary of phases $S_{1}\left(s_{\mathrm{b}}\right)=S_{2}\left(s_{\mathrm{b}}\right)=\Pi$.

\section{Numerical Results and Discussion}

\section{Importance of curvature distribution}

In the following, the effect of SC distribution on shape transformation of vesicle membranes is compared. Surveying these cases reveals that the different distributions of SC have notable effects on the final shape of the vesicle.

In Figures 2 and 3, curvature distribution function in phase 2 is presented beside each case (left figure in each case). Furthermore, the amount of SC in phase 2 is displayed schematically as a dotted line at each point of the surface perpendicular to the membrane above the initial configuration of the vesicle (middle figure in each case). In this depiction, for outward curvature the value supposed to be positive. As the dotted line gets further from the surface, the SC value is greater. The last shape on the left is the final vesicle form obtained from the simulation.

The comparison of different gradients of SC distribution and constant SC, as examples, is shown in Figure 2. Curvature gradient size reflects curvature inducer concentration on the membrane surface. Initial conditions are assumed to relate to an oblate vesicle with $x^{(1)}=0.75$ and $\hat{C}^{(i)}=0$. SC of phase 2 has a constant value $\hat{C}^{(2)}=0.4$ (Figure $2 \mathrm{~d}$ ). The parameter $a$ reveals the slope of the curvature changes with geometry which is equal to 1, 3 and 5, from Figure $2 \mathrm{a}-2 \mathrm{c}$ respectively. Except in Figure 2d, about half of the phase 2 is under the influence of SC, meaning that $b=a / 2$. 

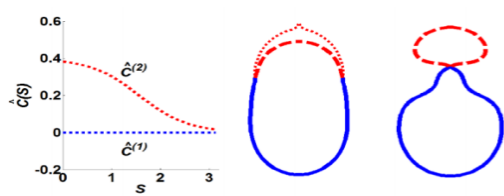

a
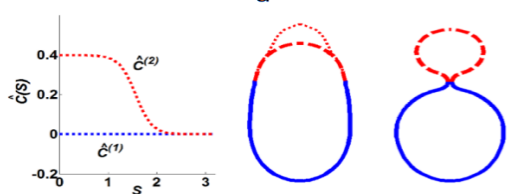

b
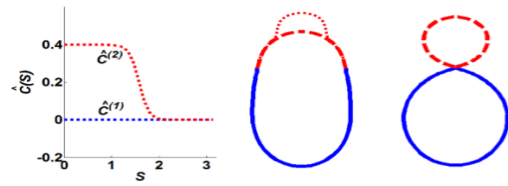

c
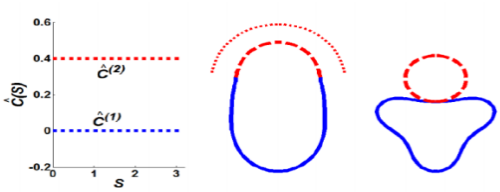

d

Figure 2: The effect of variable SC with different gradients. In all cases $\mathrm{x}^{\wedge}((1))=0.75$ and $\mathrm{C}^{\wedge} \wedge((1))=0$. a) $\left.A=0.2, a=1, b=0.5, c=0, b\right) A=0.2, a=3$, $b=1.5, c=0, c) A=0.2, a=5, b=2.5, c=0$ d) $C^{\wedge \wedge}((2))=0.4$.
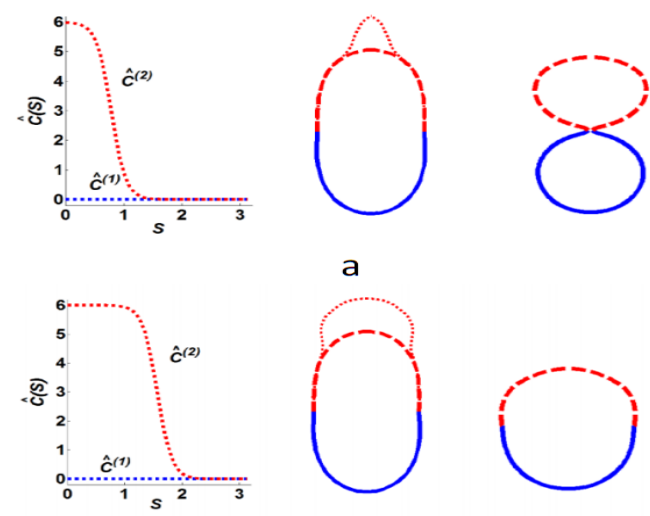

b
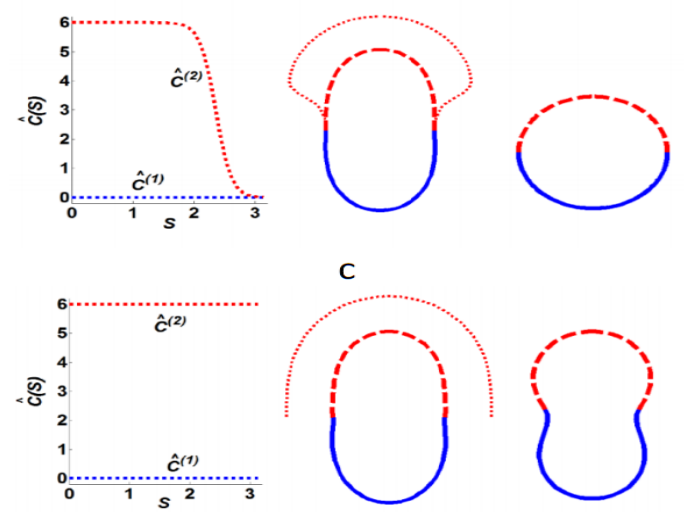

d

Figure 3: Different distribution of SC with diverse surface coverage. In all cases $\mathrm{x}^{\wedge}((1))=0.50$ and $\mathrm{C}^{\wedge}((1))=0$. a) $\mathrm{A}=3, \mathrm{a}=4, \mathrm{~b}=1, \mathrm{C}=0$, a) $\mathrm{A}=3, \mathrm{a}=4, \mathrm{~b}=2$, $c=0, c) A=3, a=4, b=3, c=0$ d) $C^{\wedge}((2))=6$.
In Figure 2a, a relatively moderate change in curvature makes phase 1 in the vicinity of the boundary to have more positive curvature, under the influence of the phase 2 SC. But in Figure $2 \mathrm{c}$ and $2 \mathrm{~d}$ there is a step change in SC distribution, not an induced membrane curvature close to the domain boundary, and therefore there is not a significant positive curvature in this area. The vesicle shape under the effect of variable SC is dissimilar to that observed with a uniform SC. These differences can be seen in overall shape and also details of vesicle structure (such as the neck geometry).

In Figure 2a, under the influence of the phase 2 SC, phase 1 in the vicinity of the boundary is impressed by a positive curvature. But in Figure $2 \mathrm{~b}$ and $2 \mathrm{c}$ there is a step change in SC distribution of phase 2 which does not induce any curvature in the membrane close to the domain boundary. Since the phase $1 \mathrm{SC}$ is zero, $\left(\hat{C}^{(i)}=0\right)$, thus, there is not a significant positive curvature in the phase 1 near the boundary. The vesicle shape under the effect of variable SC is dissimilar to the one observed with a uniform SC. These differences can be seen in overall shape and also in details of vesicle structure (such as the neck geometry) (Figure 3).

In another example, the different bending stiffness is considered $\left(k^{(2)}=4 k^{(1)}\right)$ (Figure 4). The curvature signs are opposite in the domains and the negative SC is uniformly distributed in the phase 2 ( $\left.\hat{C}^{(2)}=-3\right)$. The SC of the phase 1 is positive and the affected region of this phase by the SC is gradually increased, from Figure 4a-4d respectively. As can be seen, if the spontaneous curvature of phase 1 is over than 0.75 of the phase 1 area, it would be possible to fission of a single-phase vesicle from the complete bud.

Shape transformation depends on the occupied area with a given SC, as investigated in Figure 3. It is assumed that $x^{(1)}=0.50, \hat{C}^{(1)}=0$ and a non-uniform SC is considered only in phase 2. In these examples, for the specified curvature value and gradient, $\mathrm{A}=6$ and $\mathrm{a}=4$, and the fraction of the phase 2 area which covered with SC, is $0.25,0.50$ and 0.75 from Figure $3 a-3 c$, respectively. When SC is extended over less than half of Phase 2, the vesicle obtains a dumbbell form; but with a wider curvature distribution the vesicle shape is more nearly spherical. Applying constant curvature, the vesicle experiences local deformation only at the domain boundary (Figure 3d). Regarding the zero SC in phase 1 , in the border phases the membrane shape follows the curvature of phase 2. Evaluating these figures we find that to achieve the correct solution in any transformation process requires accurate modeling of the curvature inducing factor.

\section{Pearling}

A pearling phenomenon is one of the vesicle configurations that occur due to SC [16]. Polymers can change the lipid bilayer local SC by permeation and anchorage on the membrane leading to significant shape transformations in the vesicle [17-19]. Using the model presented here, we simulated pearling of a giant vesicle's membrane. The initial vesicle shape seems to be an oblate lipid vesicle and area fraction parameter of the phase 1 is equal to $x^{(1)}=0.98$. Physical characteristics of the vesicle membrane are assumed to be uniform in total surface and the bending rigidity of two phases are equal. It is assumed that polymer permeation through the membrane makes for a strong curvature in a small area fraction (about 2\%) and that the surrounding areas are narrowly affected by curvature induction. Accordingly, curvature distribution in 2 phases is considered as follows:

$$
\begin{aligned}
& \hat{C}^{(1)}=-2\left(1-\tanh \left(5 S_{1}-5 \pi\right)\right)+5 \\
& \hat{C}^{(2)}=2\left(1-\tanh \left(5 S_{2}-5 \pi\right)\right)+4
\end{aligned}
$$



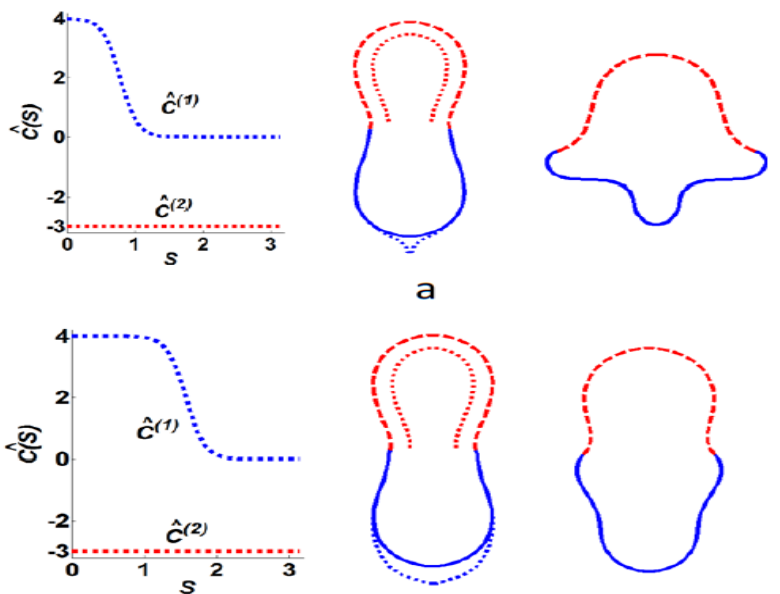

a
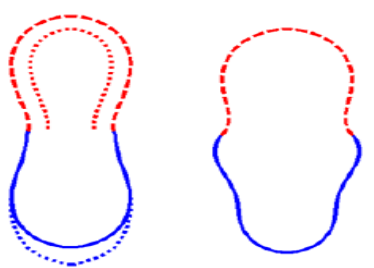

b
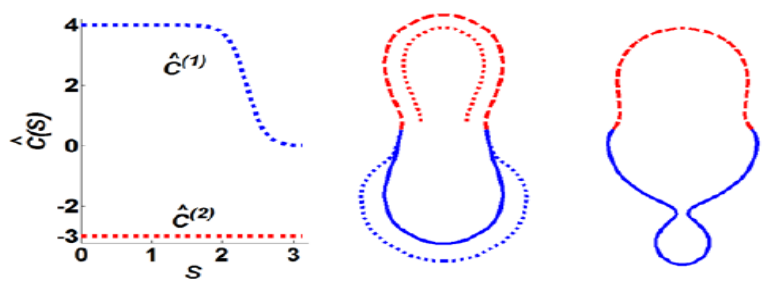

C
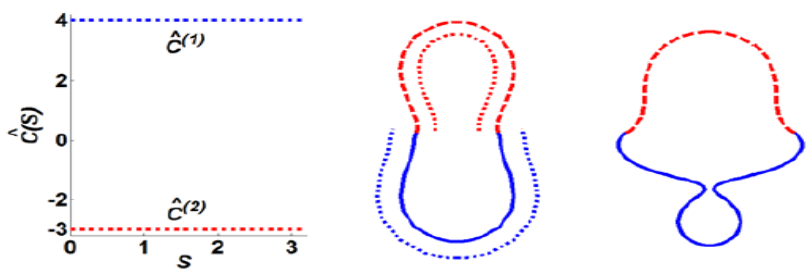

d

Figure 4: The effect of variable SC in two-component vesicle with $k^{(2)}=4 k^{(1)}$. In all cases $x^{(1)}=0.50$ and $C^{(2)}=-3$. a) $\left.\left.A=2, a=4, b=1, c=0, b\right) A=2, a=4, b=2, c=0, c\right)$ $A=2, a=4, b=3, c=0, d) C^{(1)}=4$.

Figure 5a shows curvature distribution in phases in two ways: curvature changing in each phase, $\hat{C}^{(i)}\left(S_{i}\right)$, for a central angle of $0 \leq S_{i} \leq \pi$, and schematic comparative representation of curvature distribution in which the curvature value of each point on the membrane surface is shown as a vertical distance from the membrane.

The result of the local shape transformation simulation of a giant vesicle in budding, tubulation and pearling stages is displayed in Figure $5 \mathrm{~b}$. The same stages in pearling have also been observed experimentally, as reported by others [16]: an oblate initial shape for the giant vesicle, bud-tube shapes developed on the vesicle surface, and finally pearling of the membrane. The results from these experimental data and our simulation are in good conformity with each other. In various simulations that have been done with different values of SC, it reveals that the stronger the SC, the more the process of shape transformation makes progress from budding to pearling. This phenomenon is mentioned in Ref. [17] as well.

Based on the definition of mean curvature $\left(H=\frac{1}{R_{1}}+\frac{1}{R_{2}}\right)$ and regarding the equation for a membrane's bending energy (1), for developing cylinder with radius of $R_{C}=1 / C_{0}$, and connected spheres (like the beads of necklace) (Figure 6) with radius of $R_{s}=2$ / $C_{0}$, it consumes no energy if SC is $C_{0}$. Due to the diversity between

the radius of the cylindrical tube and spheroid pearls, the geometry should be transformed smoothly. This conversion takes place with an intermediate shape that is called unduloid and converts the tube radius to the sphere radius or to zero (in the connectivity region) [19]. All of these three shapes have been observed in the simulation (Figure 5). It should be noted that in the last state, mean curvature of spheres has increased by about $50 \%$ from the curvature of cylindrical tube area leading to a reduction of approximately $30 \%$ in radius. It shows that the induced curvature in cylindrical tube is not proportional to level of membrane's energy resulting from applying SC, and for increasing curvature, the membrane inclined to spherical shape. In this regard, it is necessary to point to similarity of the curvature of vesicle's main body which has an almost constant level of energy during the process $[20,21]$.

Because of similarity of SC values in corresponding phases, the effect of variable or fixed SC can be observed through comparing Figures 5 and 7. In Figure 7a, SC is assumed to be constantly distributed in two phases and its value is $\hat{C}^{(1)}=1$ and $\hat{C}^{(2)}=8$. Due to the significant difference between the two phases, shape transformation is limited to phase 2 and because of the lack of a shape transformation in phase 1, only a bud is developed in phase 2 , which becomes gradually smaller (Figure $7 b$ ). The difference of between the conditions giving rise to Figures 5 and 7 is the curvature value in the vicinity of the phase's border. As mentioned before (Figure 5a), the curvature of phases come close to each other gradually at their border. For this reason, as SC increases at the vicinity of the border in phase 1 along with budding in phase 2, shape transformation in phase 2 leads to transformation of a bud to a tube and then to pearled sphere.

\section{Conclusions}

Due to various mechanisms of inducing SC in membranes, nearing real-life conditions for this parameter, for example nonuniform distribution, can make for a better understanding of the subject. In the current study, a model of a two-phase vesicle with varying distribution of SC is developed which relies on conventional
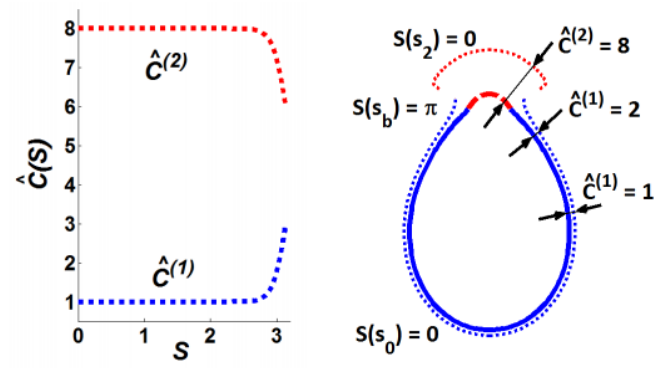

a

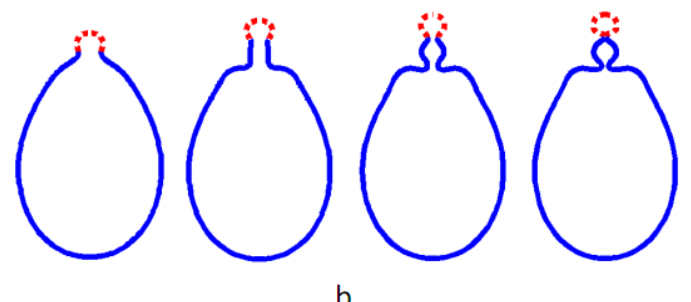

Figure 5: a) Curvature distribution in $C(S)-S$ plane (left) and schematic curvature distribution in each phase perpendicular to the membrane (right $C^{(1)}=-2\left(1-\tanh \left(5 S_{1}-5 \Pi\right)\right)+5, \quad C^{(2)}=2\left(1-\tanh \left(5 S_{2}-5 \Pi\right)\right)+4$. b- Simulating polymer permeation through the membrane and developing bud-tube shape and pearled spheres (from left to right). 


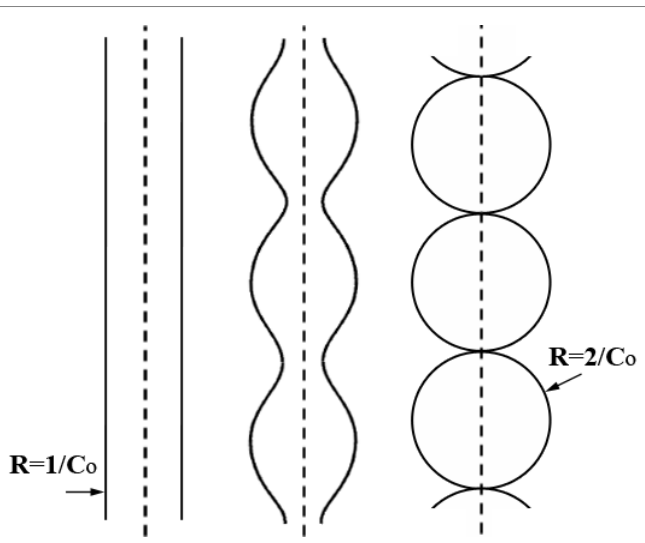

Figure 6: Cylinder and necklace-shaped spheres and unduloid transition state having a similar local mean curvature.
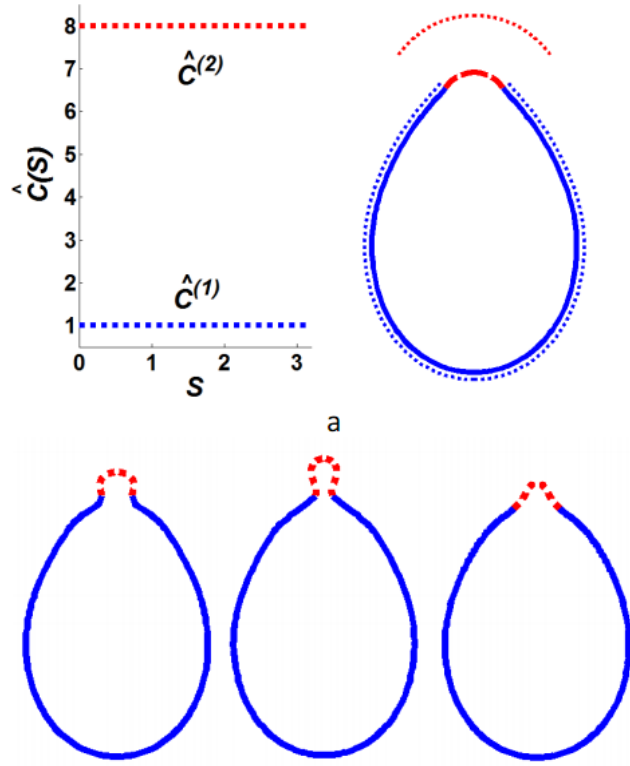

b

Figure 7: Evolution of giant vesicle's membrane due to constant SC in two phases. a) Representation of curvature function and distribution on membrane initial configuration $\left(C^{(1)}=1, C^{(2)}=8\right)$. b) Result of simulation: Generated bud gradually becomes smaller (from left to right).

continuum theory of lipid membranes. Non-uniform SC is considered as a function of arc length in each phase. In this way, the effect of different origins of SC like type and distribution of proteins and changes in solution density can be introduced in the model vesicle. Regarding each SC mechanism, a function can be introduced to represent the curvature distribution that considers the properties of the mechanism by determination of magnitude, gradient and affected area. As previously stated, the curvature distribution of a mechanism (protein, concentration, etc.), is the required input which can be calculated using laboratory test or molecular dynamics calculation. However, the challenge of this method is defining the curvature distribution which needs to be characterized either by molecular calculation or experiment. The shape transformation of a giant vesicle introduced by SC in two phases, and the simulation of bud, tube and pearl-shaped sphere are simulated by this model. It has also been shown that constant values and variable distributions of SC affect the final shape of a vesicle.

\section{Conflict of Interest}

The authors declare that they have no conflict of interest.

\section{References}

1. Kukulski W, Schorb M, Kaksonen M, Briggs JAG (2012) Plasma membrane reshaping during endocytosis is revealed by time-resolved electron tomography. Cell 150: 508-520.

2. Arkhipov A, Yin Y, Schulten K (2008) Four-scale description of membrane sculpting by BAR domains. Biophysical Journal 95: 2806-2821.

3. Dobereiner HG, Selchow O, Lipowsky R (1999) Spontaneous curvature of fluid vesicles induced by trans-bilayer sugar asymmetry. European Biophysics Journal 28: 174-178.

4. Wang $\mathrm{H}$, Hu D, Zhang $P$ (2013) Measuring the spontaneous curvature of bilayer membranes by molecular dynamics simulations. Communications in Computational Physics 13: 1093-1106.

5. Helfrich W (1973) Elastic properties of lipid bilayers: theory and possible experiments. Zeitschrift für Naturforschung, pp: 1865-7125.

6. Julicher F, Lipowsky R (1996) Shape transformations of vesicles with intramembrane domains. Physical Review E 53: 2670.

7. Frese RN, Pamies JC, Olsen JD, Bahatyrova S, van der Weij-de CD, et al. (2008) Protein shape and crowding drive domain formation and curvature in biological membranes. Biophysical Journal 94: 640-647.

8. West B, Schmid F (2010) Membrane-Protein Interactions in Lipid Bilayers: Molecular Simulation versus Elastic Theory. Forschungszentrum Julich 3: 279

9. Agrawal A, Steigmann DJ (2009) Modeling protein-mediated morphology in bio membranes. Biomechanics and Modeling in Mechano Biology 8: 371-379.

10. Rangamani $P$, Mandadap KK, Oster G (2014) Protein-induced membrane curvature alters local membrane tension. Biophysical Journal 107: 751-762.

11. Campelo F, Hernández-Machado A (2008) Polymer-induced tubulation in lipid vesicles. Physical Review Letters 100: 158103.

12. Rahimi M, Arroyo M (2012) Shape dynamics, lipid hydrodynamics, and the complex viscoelasticity of bilayer membranes. Physical Review E 86: 011932.

13. Sahebifard M, Shahidi A, Ziaei-Rad S (2016) Two-phase vesicles: a study on evolutionary and stationary models. European Biophysics Journal, pp: 1-8.

14. Baumgart T, Das S, Webb W, Jenkins J (2005) Membrane elasticity in giant vesicles with fluid phase coexistence. Biophysical Journal 89: 1067-1080.

15. Boal D (2002) Mechanics of the cell Cambridge. Cambridge University Press, UK

16. Tsafrir I, Caspi Y, Guedeau-Boudeville MA, Arzi T, Stavans J (2003) Budding and tubulation in highly oblate vesicles by anchored amphiphilic molecules. Physical Review Letters 91: 138102.

17. Bickel T, Jeppesen C, Marques CM (2001) Local entropic effects of polymers grafted to soft interfaces. The European Physical Journal E 4: 33-43.

18. Lipowsky R, Döbereiner HG, Hiergeist C, Indrani V (1998) Membrane curvature induced by polymers and colloids. Physica A: Statistical Mechanics and its Applications 249: 536-543.

19. Seifert U, Berndl K, Lipowsky R (1991) Shape transformations of vesicles: Phase diagram for spontaneous-curvature and bilayer-coupling models. Physical Review A 44: 1182.

20. Marsh D (2007) Lateral pressure profile, spontaneous curvature frustration, and the incorporation and conformation of proteins in membranes. Biophysical Journal 93: 3884-3899.

21. Seifert U (1997) Configurations of fluid membranes and vesicles. Advances in Physics 46: 13-137. 